\title{
Chronic Kidney Disease-Mineral and Bone Disorder KDIGO Guidelines
}

\author{
A. Khwaja \\ Sheffield Kidney Institute, Northern General Hospital, Sheffield, UK
}

2009 saw the publication of the clinical practice guidelines from Kidney Disease Improving Global Outcomes (KDIGO) relating to diagnosis, evaluation, prevention and treatment of chronic kidney disease-mineral and bone disorder (CKD-MBD) [1].

KDIGO has utilised a grading system for clinical practice recommendations with the strength of recommendation being graded as level 1 or level 2, with level 1 being a 'we recommend ...' statement implying most patients should receive this action whilst level 2 is a 'suggestion' recognising that management decisions may vary in different clinical contexts. Each recommendation was further graded from A to D by the quality of evidence underpinning them, with grade A referring to a high quality of evidence whilst grade D recognised a 'very low' evidence base.

KDIGO defined CKD-MBD as systemic disorder of mineral and bone metabolism due to CKD manifested by either one or a combination of the following:

- abnormalities of calcium, phosphate, parathyroid hormone (PTH) or vitamin D metabolism,

- abnormalities in bone turnover, mineralisation, volume, linear growth or strength,

- vascular or other soft-tissue calcification.

To avoid confusion renal osteodystrophy was defined as:

- an alteration of bone morphology in patients with CKD,

\section{KARGER \\ Fax +4161306 1234 E-Mail karger@karger.ch} www.karger.com
(C) 2010 S. Karger AG, Basel

$1660-2110 / 10 / 1161-0025 \$ 26.00 / 0$

Accessible online at:

www.karger.com/nec
- one measure of the skeletal component of the systemic disorder of CKD-MBD that is quantifiable by histomorphometry of bone biopsy.

The guidelines themselves focussed on three key domains relating to:

(1) diagnosis of CKD-MBD with respect to biochemical abnormalities, bone and vascular calcification,

Table 1. Diagnosis of CKD-MBD

Biochemical abnormalities

- Measure calcium, phosphate and PTH in CKD 3-5D with frequency determined degree of abnormalities and severity of CKD

- Measure 25(OH)-vitamin D (calcidiol) in CKD 3-5D

- Therapeutic decisions based on trends rather than single value and evaluate calcium and phosphate individually rather than calcium-phosphate product

Bone

- Bone biopsy if pain, unexplained fractures, hypercalcaemia, hypophosphataemia, aluminium toxicity, bisphosphonate use (no evidence grade)

- No role for routine bone mineral densitometry

- PTH/bone-specific alkaline phosphatase to evaluate bone turnover

Vascular calcification

- Detect with ECHO/lateral abdominal radiograph as valid alternative to $\mathrm{CT}$

- Calcification represents highest cardiovascular risk

Tel. +44 114271 4808, Fax +44 114243 1575, E-Mail arif.khwaja@sth.nhs.uk 
Table 2. Treatment targets for CKD-MBD

\begin{tabular}{lll}
\hline Calcium & Phosphate & PTH \\
\hline Restrict calcium if: & Goal: normal levels & CKD 3-5 \\
- Hypercalcaemic & of phosphate & treat if rising and above upper limit of normal \\
- Adynamic bone disease & & maintain $2-9 \times$ upper limit of normal; \\
- Vascular calcification & marked changes trigger treatment \\
- Low PTH & - role for calcitriol, vitamin D analogues and calcimimetics \\
& - avoid or reduce dose of vitamin D sterol/calcitriol if \\
& hypercalcaemic or hyperphosphataemic \\
\hline
\end{tabular}

Treat trends: calcium and phosphate to normal and PTH to target range.

Table 3. Evaluation and treatment of kidney transplant bone disease

Monitoring

- Calcium, phosphate, PTH, 25(OH)-vitamin D (calcidiol)

- Bone mineral density (BMD) within 3 months of transplant if eGFR $>30 \mathrm{ml} / \mathrm{min} /$

$1.73 \mathrm{~m}^{2}$ and if on steroids or other risk factors for osteoporosis

- No BMD for CKD 4-5T

Treatment

- Correct mineral abnormalities as in CKD 3-5

- Low BMD and eGFR >30 ml/min/1.73 $\mathrm{m}^{2}$ : bisphosphonates or alfacalcidol/calcitriol or vitamin $\mathrm{D}$

- Low BMD and eGFR $<30 \mathrm{ml} / \mathrm{min} / 1.73 \mathrm{~m}^{2}$ : manage as CKD 4-5

(2) treatment of phosphate, calcium, PTH and the use of bisphosphonates and growth hormone in CKD-MBD,

(3) evaluation and treatment of kidney transplant bone disease.

Key aspects of the guidelines are summarised in tables $1-3$. The guidelines represent an impressive and exhaus- tive summary of the true evidence base underlying clinical practice and have identified key gaps in knowledge. In general they are much less prescriptive than previous guidelines in this area and it is striking that KDIGO issued only 39 guideline statements in contrast to the KDOQI guidelines [2], which issued 104 guideline statements of which only 16 were evidence-based. This weak evidence base is highlighted by the fact that there were absolutely no 'A' quality studies related to patient outcomes such as mortality, hospitalisation, quality of life or clinical cardiovascular disease. To this end the group prioritised areas for future research focussing on patientcentred outcomes such as risk stratification for clinical outcomes determining the value of protocol-driven interventions for phosphate and PTH in improving hard clinical endpoints. Whilst some have complained that this is a weak and vague document leaving individual clinicians with the freedom to do almost anything (or even nothing), numerous studies remind us of the fundamental flaw and danger of basing clinical guidelines on observational data [3].

\section{References}

1 Kidney Disease: Improving Global Outcomes (KDIGO) CKD-MBD Work Group: KDIGO clinical practice guideline for the diagnosis, evaluation, prevention, and treatment of Chronic Kidney Disease-Mineral and Bone Disorder (CKD-MBD). Kidney Int Suppl 2009;113:S1-S130.

2 National Kidney Foundation: K/DOQI clinical practice guidelines for bone metabolism and disease in chronic kidney disease. Am J Kidney Dis 2003;42(4 suppl 3):S1-S201.
Pfeffer MA, Burdmann EA, Chen CY, Cooper ME, de Zeeuw D, Eckardt KU, Feyzi JM, Ivanovich P, Kewalramani R, Levey AS, Lewis EF, McGill JB, McMurray JJ, Parfrey P, Parving HH, Remuzzi G, Singh AK, Solomon SD, Toto R; TREAT Investigators: A trial of darbepoetin alfa in type 2 diabetes and chronic kidney disease. N Engl J Med 2009; 361:2019-2032. 